\title{
POLYMER MELT FLOW IN SUDDEN EXPANSIONS: THE EFFECTS OF VISCOUS HEATING
}

\begin{abstract}
P. S. B. Zdanski ${ }^{a}$,
M. Vaz. Jr. ${ }^{a}$, and A. P. C. Dias ${ }^{\mathrm{a}}$

${ }^{\text {a }}$ Universidade do Estado de Santa Catarina

Departamento de Engenharia Mecânica

Bairro Bom Retiro

CP. 89223, Joinville, Santa Catarina, Brasil zdanski@joinville.udesc.br

ABSTRACT

Sudden expansion is a typical geometry found at the entrance of a mould cavity and is generally known in industry as 'gate'. Flow simulation of this class of problems poses some difficulties owing to coupling of momentum and energy laws, existence of re-circulation and steep pressure variations. This work focuses on the physical analysis of the laminar and incompressible polymer melt flow inside channels with sudden expansions aimed at mapping the viscous heating effect. The mathematical model comprises the mass, momentum and energy conservation laws. The pressure-velocity coupling is treated on solving a Poisson equation for pressure. The Cross constitutive model is adopted to describe the nonNewtonian behavior of the flow. The governing equations are discretized using the finite difference method based on central, second order accurate formulae for both convective and diffusive terms. Artificial smoothing terms are added to control the odd-even decoupling problem. The main results demonstrate that the flow parameters, such as pressure drop along the channel and Nusselt number at the walls, are affected by viscous heating. It has been found that, in such problems, any reliable solution must account for the non-isothermal effects.
\end{abstract}

\section{NOMENCLATURE}

$c_{p} \quad$ specific heat at constant pressure, $\mathrm{J} /(\mathrm{kg} . \mathrm{K})$

$\mathrm{h} \quad$ channel thickness at the entrance, $\mathrm{m}$

$\mathrm{h}_{\mathrm{c}} \quad$ convective coefficient, $\mathrm{W} /\left(\mathrm{m}^{2} . \mathrm{K}\right)$

$\mathrm{k}$ thermal conductivity, $\mathrm{W} /(\mathrm{m} . \mathrm{K})$

$\mathrm{n}$ power-law index, non-dimensional

$\mathrm{Nu} \quad$ Nusselt number, $\mathrm{Nu}=\mathrm{h}_{\mathrm{c}} \mathrm{h} / \mathrm{k}$

$\mathrm{p}$ static pressure, $\mathrm{Pa}$

Re Reynolds number, $\operatorname{Re}=\rho \mathrm{u}_{\text {in }} \mathrm{h} / \bar{\eta}$

$\mathrm{T}$ temperature, $\mathrm{K}$

$t$ time, $s$

$\mathrm{u}_{\mathrm{i}}, \mathrm{u}_{\mathrm{j}}$ velocity components, $\mathrm{m} / \mathrm{s}$

$\mathrm{x}_{\mathrm{i}}, \mathrm{x}_{\mathrm{j}}$ Cartesian coordinates, $\mathrm{m}$

\section{Greek symbols}

$\dot{\bar{\gamma}} \quad$ equivalent shear rate, $\mathrm{s}^{-1}$

$\eta \quad$ apparent viscosity, Pa.s

$\eta_{0} \quad$ Newtonian viscosity, Pa.s

$\lambda$ material parameter, $\mathrm{s}$

$\rho \quad$ fluid density, $\mathrm{kg} / \mathrm{m}^{3}$

\section{Subscripts}

in inlet section

w wall
Keywords: non-newtonian flows, numerical analysis, sudden expansions, viscous heating

\section{INTRODUCTION}

Polymer melt flow is frequently found in many industrial applications such as injection molding processes. The numerical simulation of this class of problems has gained widespread attention in the last years. New mathematical models, realistic rheological descriptions and numerical schemes are ongoing research topics pursued by the scientific community.

In injection molding, a narrow channel is placed between the feeding channel and the mould cavity, as illustrated in Fig. 1. Such contraction is usually known in industry as gate and has, among others, the purpose of heating the melt to best fit the cavity. The heating effect is believed (empirically) to be attained due to high viscous dissipation of polymer melts. In this context, the present work aims at analyzing flow features of polymer melts inside channels with sudden expansions, specially the non-isothermal effects.

The literature in this subject is recent but still scarce, being specially devoted on studying isothermal flows. New numerical methodologies to solve the problem were proposed in Bao (2002) and Missirlis et al. (1998). The former work adopts finite elements whereas the latter uses the finite volume method, both of which within a framework of a generalized Newtonian formulation. Physical analysis of the flow bifurcation phenomenon was discussed by 
Manica and De Bortoli (2004), Neofytou and Drikakis (2003), Ternik et al. (2006) and Neofytou (2006). The references adopt the generalized Newtonian formulation with an isothermal power-law model to describe the non-Newtonian flow behavior. Pressure drop and vortex length in sudden expansions are flow features studied in Pinho et al. (2003). The authors discuss the effects of the power-law index in isothermal flows. Nitin and Chhabra (2005) present a parametric analysis of non-isothermal power-law fluid flow past a rectangular obstacle. The drag coefficient and Nusselt number are obtained as functions of Reynolds number, power law-index and Peclet number. The solution obtained by the authors corresponds to Reynolds number ranging from 5 to 40 , which are typical values for polymeric aqueous solution, but too high for polymer melts.

The present work discusses physical aspects of polymer melt flow in sudden expansions. The analysis assesses the influence of viscous heating phenomenon on flow parameters such as pressure drop along the channel and Nusselt number at the walls. The numerical scheme is based on the techniques originally conceived to solve Newtonian flows (Zdanski et al., 2004). The capability of the scheme to handle non-Newtonian flows inside plane channels was demonstrated in Zdanski and Vaz Jr. (2006a, 2006b), being straightforward its extension to solve sudden expansion flows (Vaz Jr. and Zdanski, 2007). The results obtained show that the effects of viscous heating on flow parameters are relevant as the Reynolds number increases.

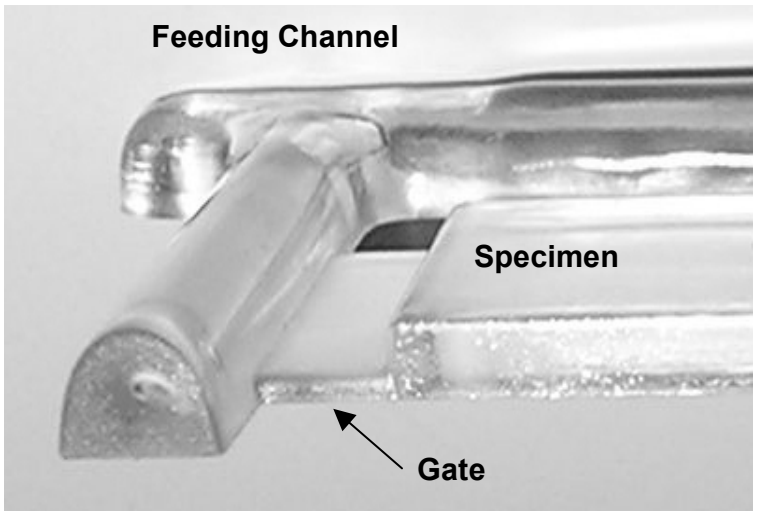

Figure 1. Typical gate as it appears in a mould cavity

\section{THEORY}

The fully coupled Navier-Stokes and energy equations, within a framework of generalized Newtonian formulation, is the mathematical model adopted, i.e.,

$$
\frac{\partial\left(\rho \mathrm{u}_{\mathrm{i}}\right)}{\partial \mathrm{t}}+\frac{\partial\left(\rho \mathrm{u}_{\mathrm{j}} \mathrm{u}_{\mathrm{i}}\right)}{\partial \mathrm{x}_{\mathrm{j}}}=-\frac{\partial \mathrm{p}}{\partial \mathrm{x}_{\mathrm{i}}}
$$

$$
\begin{aligned}
& +\frac{\partial}{\partial \mathrm{x}_{\mathrm{j}}}\left[\eta(\mathrm{T}, \dot{\bar{\gamma}})\left(\frac{\partial \mathrm{u}_{\mathrm{i}}}{\partial \mathrm{x}_{\mathrm{j}}}+\frac{\partial \mathrm{u}_{\mathrm{j}}}{\partial \mathrm{x}_{\mathrm{i}}}\right)\right] \\
& \frac{\partial\left(\rho \mathrm{c}_{\mathrm{p}} \mathrm{T}\right)}{\partial \mathrm{t}}+\frac{\partial\left(\rho \mathrm{c}_{\mathrm{p}} \mathrm{u}_{\mathrm{i}} \mathrm{T}\right)}{\partial \mathrm{x}_{\mathrm{i}}}=\frac{\partial}{\partial \mathrm{x}_{\mathrm{i}}}\left(\mathrm{k} \frac{\partial \mathrm{T}}{\partial \mathrm{x}_{\mathrm{i}}}\right)+\eta(\mathrm{T}, \dot{\bar{\gamma}}) \dot{\bar{\gamma}}^{2},
\end{aligned}
$$

where $\eta$ is the apparent viscosity, $\mathrm{Pa} / \mathrm{s}^{-1}, \mathrm{~T}$ the temperature, $\mathrm{K}$, and $\dot{\bar{\gamma}}$ the equivalent shear rate, $\mathrm{s}^{-1}$. The remaining symbols in the preceding equations are completely standardized in literature and given by: $\mathrm{p}$ is the pressure, $\mathrm{Pa} ; \rho$ the fluid density, $\mathrm{kg} / \mathrm{m}^{3} ; \mathrm{u}_{\mathrm{i}}$ and $\mathrm{u}_{\mathrm{j}}$ the velocity components, $\mathrm{m} / \mathrm{s} ; \mathrm{c}_{\mathrm{p}}$ the specific heat at constant pressure, $\mathrm{J} / \mathrm{kgK} ; \mathrm{x}_{\mathrm{i}}$ and $\mathrm{x}_{\mathrm{j}}$ the Cartesian coordinates, $\mathrm{m}$; $\mathrm{t}$ the time, $\mathrm{s}$.

The simulations performed in this work are for a commercial polymer Polyacetal POM-M90-44, whose non-Newtonian behavior is accounted for the Cross constitutive model as

$$
\eta(\mathrm{T}, \dot{\bar{\gamma}})=\frac{\eta_{0}}{1.0+[\lambda(\mathrm{T}) \dot{\bar{\gamma}}]^{1.0-\mathrm{n}(\mathrm{T})}}
$$

in which $\eta_{0}$ is the Newtonian viscosity, $\mathrm{Pa} / \mathrm{s}^{-1}, \mathrm{n}$ is the power-law index, non-dimensional, and $\lambda$ is a material parameter, $s$, whose temperature dependence is expressed by the Arrhenius law. For more details about the governing equations and constitutive model the reader is referred to Zdanski and Vaz Jr. (2006a, 2006b). The incompressible flow approach employed to derive the numerical approximation requires use of a pressure-velocity coupling scheme. The method used in this work follows the same route presented in Zdanski et al. (2004), where a Poisson equation for pressure is solved to assure divergence-free velocity field.

\section{NUMERICAL METHOD}

As the emphasis of the present work is the physical analyses of important aspects of polymer melt flow in sudden expansions, only a brief discussion on the numerical method is given. Central finite difference formulae were used to discretize both convection and diffusion terms of the governing equations. Therefore, the scheme is second order accurate in space. The variables are arranged in a colocated mesh, and artificial viscosity terms are added externally to control the odd-even decoupling problem. The method is implicit and follows a pseudo-transient march in time, aiming at achieving the steady state solution. The present methodology was originally proposed to solve Newtonian flows (Zdanski et al., 2004), and has been successfully applied to non-Newtonian flows by Zdanski and Vaz Jr. (2006a, 2006b) and Vaz Jr. and Zdanski (2007). 


\section{RESULTS AND DISCUSSION}

The non-Newtonian flow in sudden expansions, despite the simplicity of the geometry, exhibits some complex fluid flow behavior. The presence of recirculation regions and steep property variations impose difficulties leading to a demanding computational case. In the practical sense, a flow expansion is typically found at the 'gate' and its physical understanding is important in industrial injection molding processes. Figure 1 represents a typical 'gate' located at the entrance of a mould cavity. The expansion section can be clearly seen and the problem under discussion is of great interest in the practical sense as well as in a scientific ground.

The geometry simulated in the present work is the 1:2 asymmetric expansion, being the channel length $3 \mathrm{~h}$ and $20 \mathrm{~h}$ upstream and downstream of the expansion section respectively ( $h$ is the channel thickness of the entrance section and is assumed $\mathrm{h}=$ $4 \mathrm{~mm})$. The geometry, with its main dimensions, is represented at Fig. 2. The computational mesh adopted is non-uniform with points clustering in the regions near the corner and solid walls. A total of 41 x 81 and 151 x 161 grid points are used for mapping the channel regions upstream and downstream of the expansion section, respectively. The boundary conditions used in the simulations are the following: no-slip condition for velocity components and prescribed temperature at solid walls; uniform velocity and temperature profiles at the entrance and parabolic extrapolation (non-reflexive) at the exit section; for pressure, a linear variation is imposed at the entrance section whereas the parabolic condition is enforced at both solid walls and exit section.

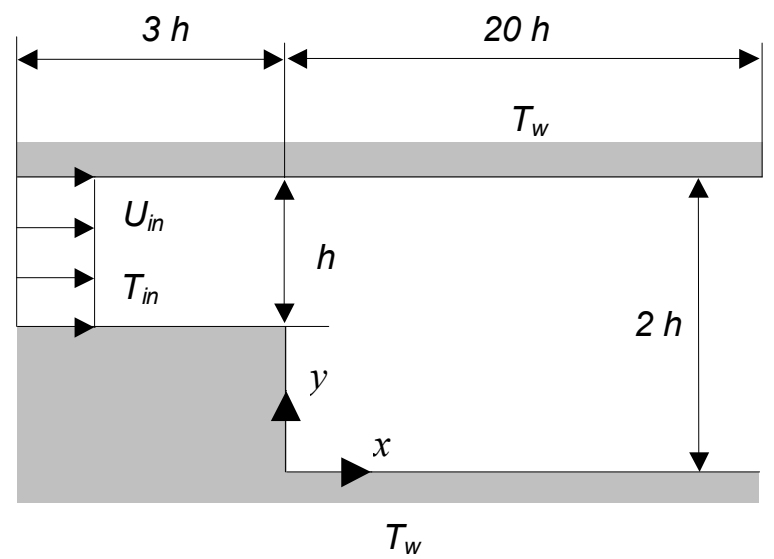

Figure 2. Sudden expansion geometry with its main dimensions

The aim of the present work is to investigate the influence of viscous heating on expansion flows. The inlet velocity, $u_{i n}$, is varied from $4 \mathrm{~cm} / \mathrm{s}$ to $50 \mathrm{~cm} / \mathrm{s}$. This velocity range renders Reynolds numbers around $10^{-4} \sim 10^{-3}$. The temperatures enforced at walls and inlet section correspond to two set of values, i.e,
$\mathrm{T}_{\mathrm{w}}=\mathrm{T}_{\mathrm{in}}=453.0 \mathrm{~K}$ and $\mathrm{T}_{\mathrm{in}}=473.0 \mathrm{~K}>\mathrm{T}_{\mathrm{w}}=453.0 \mathrm{~K}$. The following thermal problems are addressed: simulation using (i) the complete energy equation as it appears in Eq. (2), and (ii) the energy equation without viscous dissipation (without the last term of Eq. (2)). The preceding strategy aims at assessing the influences of viscous heating in such flows for the present Reynolds number range.

Some general features of the flow topology are presented for $\mathrm{T}_{\text {in }}(473.0 \mathrm{~K})>\mathrm{T}_{\mathrm{w}}(453.0 \mathrm{~K})$ in Figs. $3-$ 6 . The results correspond to the solution of thermal problems (i) and (ii) where inlet velocity is set constant $u_{i n}=10 \mathrm{~cm} / \mathrm{s}$. Streamlines at the neighborhood of the step are represented at Fig. 3, in which, a small vortex zone appears in the region at the corner. The bubble is extremely confined at the concave corner due to the high fluid viscosity of polymer melts. This flow topology is qualitatively in accordance with Bao's results for isothermal flows (Bao, 2003). The re-circulation length is approximately $\mathrm{x} / \mathrm{h}=0.25$. It is interesting to mention that the flow topology is very similar for both thermal problems (i) and (ii), being the differences in the vortex length virtually negligible. This feature is credited to the extremely low Reynolds number of the problem.

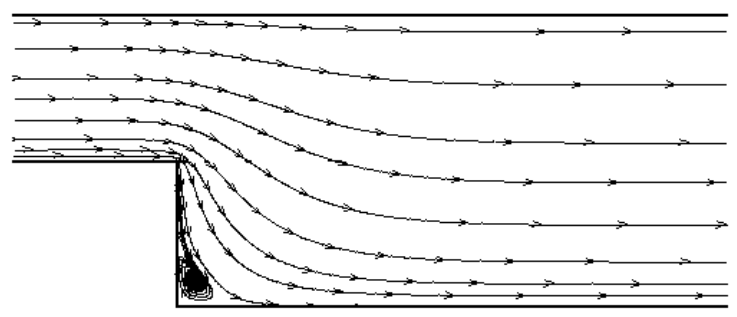

Figure 3. Streamlines at the expansion region for thermal problem (i)

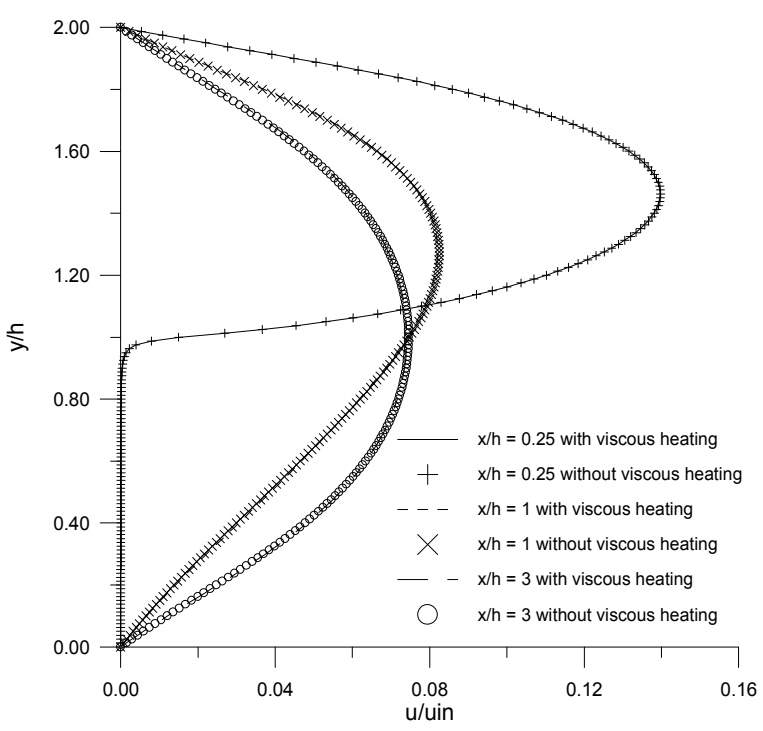

Figure 4. Velocity profiles from $\mathrm{y} / \mathrm{h}=0$ (lower wall) to $\mathrm{y} / \mathrm{h}=2$ (upper wall) 
Velocity, Temperature and viscosity profiles at selected stations $\mathrm{x} / \mathrm{h}$ (where $\mathrm{x}$ is measured from the expansion section) are plotted in order to evaluate the differences between two thermal problems simulated. The attention is focused on the neighborhood of the expansion section, e.g., $\mathrm{x} / \mathrm{h}=0.25 ; 1.00 ; 3.00$. The profiles shown at Figs. $4-6$ highlight that viscous heating has little effect on the property distributions for inlet velocities lower than $10 \mathrm{~cm} / \mathrm{s}\left(\mathrm{u}_{\text {in }} \leq 10 \mathrm{~cm} / \mathrm{s}\right.$ and $\mathrm{h}=4 \mathrm{~mm}$ ). It has been found that viscous heating promotes a maximum temperature rise of $0.9 \mathrm{~K}$ for the conditions simulated (around $\mathrm{y} / \mathrm{h}=0.25$ for $\mathrm{x} / \mathrm{h}=$ 1.0). Further discussions regarding the shape of the profiles are addressed in authors' previous works for plane channels (Zdanski and Vaz Jr. 2006a, 2006b) and expansion flows (Vaz Jr. and Zdanski, 2007).

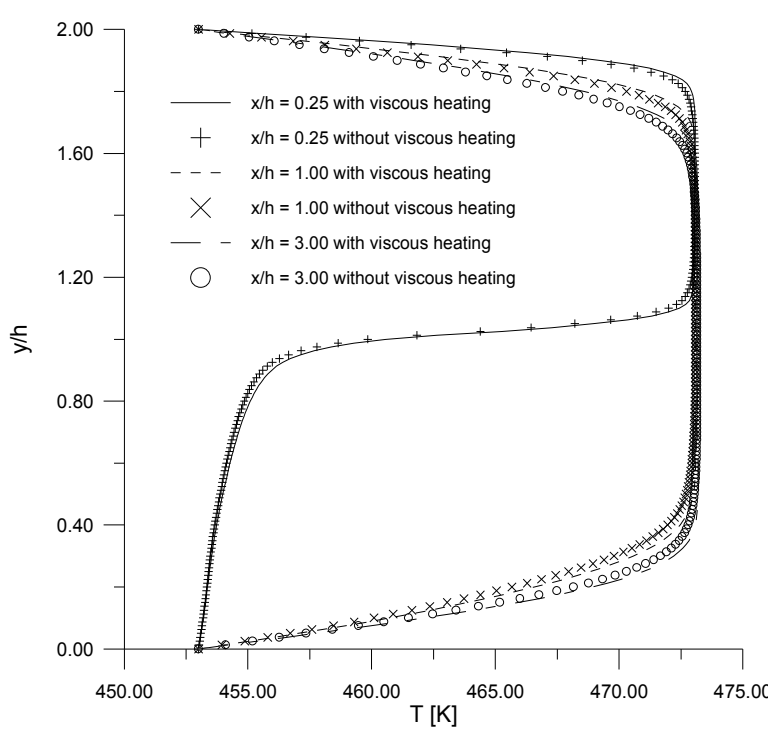

Figure 5. Temperature profiles from $\mathrm{y} / \mathrm{h}=0$ (lower wall) to $\mathrm{y} / \mathrm{h}=2$ (upper wall)

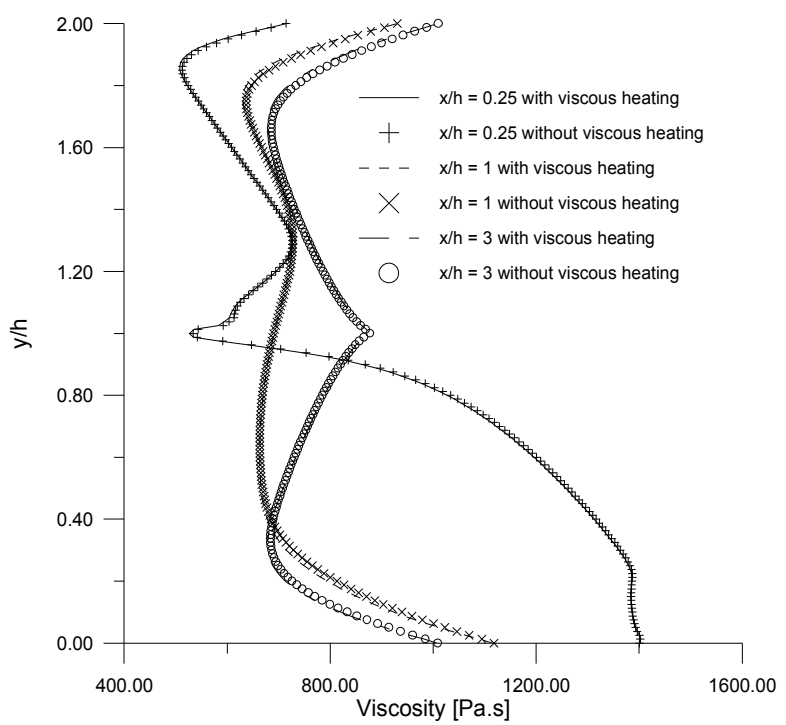

Figure 6 . Viscosity profiles from $\mathrm{y} / \mathrm{h}=0$ (lower wall) to $\mathrm{y} / \mathrm{h}=2$ (upper wall)
The mean pressure drop from inlet to exit channel sections for the two thermal problems simulated and different inlet velocities is shown in Fig. 7. The temperatures adopted as boundary condition at entrance section and wall are $\mathrm{T}_{\text {in }}=\mathrm{T}_{\mathrm{w}}=$ $453.0 \mathrm{~K}$ and $\mathrm{T}_{\text {in }}=473.0 \mathrm{~K}>\mathrm{T}_{\mathrm{w}}=453.0 \mathrm{~K}$. The results are presented as function of Reynolds number,

$\operatorname{Re}=\frac{\rho \mathrm{u}_{\mathrm{in}} \mathrm{h}}{\bar{\eta}}$,

where the mean viscosity $(\bar{\eta})$ is estimated at the channel exit section. Clearly, the figure shows that viscous heating effect is getting more pronounced as velocities are increased (higher Reynolds numbers). For lower velocities $\left(u_{i n}<20 \mathrm{~cm} / \mathrm{s}\right)$, the maximum difference of pressure losses is $1 \%$ for thermal problems (i) and (ii). Besides, one assesses that pressure drop is ever lower for thermal problem (i), especially at higher Reynolds number. This is a consistent result since at higher Reynolds number viscous heating promotes an overheating causing the viscosity to decrease rendering lower pressure losses. When comparing the solutions for the two sets of inlet temperatures adopted one assesses a reduction of around 34\% in pressure losses for higher Reynolds number when inlet temperature is raised by $20 \mathrm{~K}$. This result demonstrates the great influence of temperature on pressure variations and consequently on power required to pump the polymer inside a mould cavity.

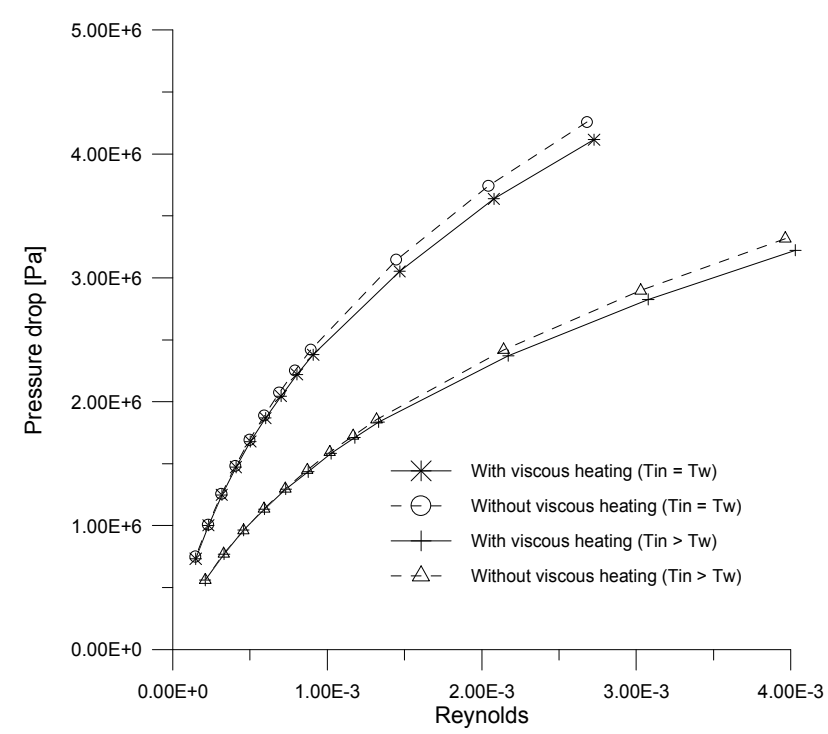

Figure 7. Mean pressure drop from inlet to exit channel sections

The local Nusselt number distributions for thermal problems (i) and (ii) are presented at Fig. 8. The following Nusselt definition is adopted,

$\mathrm{Nu}=\frac{\mathrm{h}_{\mathrm{c}} \mathrm{h}}{\mathrm{k}}$, 
where the convective coefficient is defined as

$$
\mathrm{h}_{\mathrm{c}}=\frac{\mathrm{q}^{\prime \prime}}{\left(\mathrm{T}_{\mathrm{w}}-\mathrm{T}_{\mathrm{m}}\right)} \text {. }
$$

In the preceding expression $T_{m}$ is the classical bulk temperature and $\mathrm{q}_{\mathrm{s}}$, the heat flux evaluated at the lower wall $(\mathrm{y} / \mathrm{h}=0)$. The results correspond to inlet temperature $\mathrm{T}_{\text {in }}=473.0 \mathrm{~K}>\mathrm{T}_{\mathrm{w}}=453.0 \mathrm{~K}$ for two distinct inlet velocities $u_{i n}=6 \mathrm{~cm} / \mathrm{s}$ and $u_{\text {in }}=18 \mathrm{~cm} / \mathrm{s}$. Once again the results clearly evince that viscous heating becomes more important for increasing velocities. Noticeably, for inlet velocity $6 \mathrm{~cm} / \mathrm{s}$, the Nusselt number for problem (i) is around 4\% higher than problem (ii) (for the range $\mathrm{x} / \mathrm{h}>2.0$ ). Otherwise, when inlet velocity is raised to $\mathrm{u}_{\mathrm{in}}=18 \mathrm{~cm} / \mathrm{s}$, the differences between the two thermal problems become around $15 \%$ (for $\mathrm{x} / \mathrm{h}>2.0$ ). Besides, the Nusselt number is always higher when viscous heating is considered, an expected result. It is interesting to note that the general trend of the Nusselt curve for the present polymer is similar to the Newtonian flow in sudden expansions (Zdanski at al., 2005), i.e., there is a local maximum near the reattachment point, followed by a slow decrease as the flow recovers the plane channel tendency.

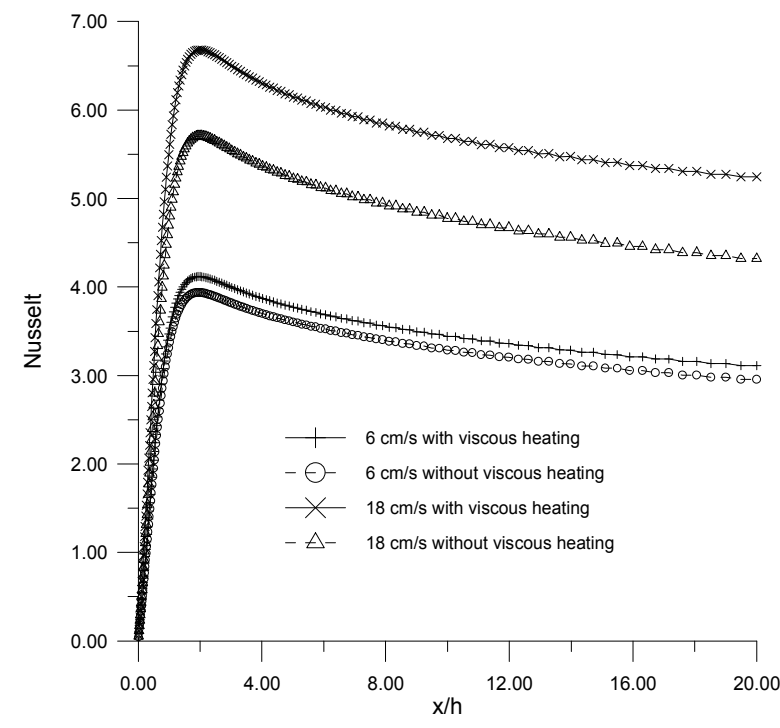

Figure 8. Nusselt number distribution along the channel length

\section{CONCLUSIONS}

The Polymer melt flow in sudden expansions is studied numerically. The generalized Newtonian formulation is adopted, being the non-Newtonian behavior of the flow described by the Cross constitutive relation. The numerical method was able to handle successfully the strong non-linearity and coupled character of the problem. The results obtained clearly evince the effects of viscous heating on pressure losses and Nusselt number for this class of flow. The findings may be summarized as follows: (a) it has been found that viscous heating promotes a maximum temperature rise of $0.9 \mathrm{~K}$ for inlet velocities smaller than $10 \mathrm{~cm} / \mathrm{s}$; it is worth to mention that the range of velocities simulated in this work is typically found in industrial injection problems; therefore, the findings may be relevant for technological field; (b) mean pressure drop in the channel is strongly affected by the inlet temperature; otherwise, the viscous heating exhibit only moderate influence; for the range simulated $\left(u_{i n}<50 \mathrm{~cm} / \mathrm{s}\right)$, the maximum difference of pressure losses is found to be $4 \%$ for thermal problems (i) and (ii); in addition, an inlet temperature rise of $20 \mathrm{~K}$ leads to a reduction in the mean pressure drop of around $34 \%$ for both thermal problems; (c) the Nusselt number is more sensitive to the viscous heating effect than to pressure losses; when inlet velocity is increased to $18 \mathrm{~cm} / \mathrm{s}$ the difference between two thermal problems becomes $15 \%$.

\section{ACKNOWLEDGEMENTS}

The co-author A. P. C. Dias gratefully acknowledges the $\mathrm{PIBIC} / \mathrm{CNPq} / \mathrm{UDESC}$ financial support program.

\section{REFERENCES}

Bao, W., 2003, An economical finite element aproximation of generalized Newtonian flows, Computer Methods in Applied Mechanics and Engineering, Vol. 191, pp. 3637-3648.

Koh, Y. H., Ong, N. S., Chen, X. Y., Lam, Y. C. and Chai, J.C., 2004, Effect of temperature and inlet velocity on the flow of a non-Newtonian fluid, International Communications in Heat and Mass Transfer, Vol. 31, No. 7, pp. 1005-1013.

Neofytou, P., 2006, Transition to asymmetry of generalized Newtonian fluid flows through a symmetric sudden expansion, Journal of NonNewtonian Fluid Mechanics, Vol. 133, pp. 132-140.

Neofytou, P. and Drikakis, D., 2003, NonNewtonian flow instability in a channel with a sudden expansion, Journal of Non-Newtonian Fluid Mechanics, Vol. 111, pp. 127-150.

Nitin, S. and Chhabra, R. P., 2005, Nonisothermal flow of a power law fluid past a rectangular obstacle (of aspect ratio $1 \times 2$ ) in a channel: Drag and heat transfer, International Journal of Engineering Science, Vol. 43, pp. 707-720.

Manica, R., and De bortoli, A. L., 2004, Simulation of sudden expansion flows for power law fluids, Journal of Non-Newtonian Fluid Mechanics, Vol. 121, pp. 35-40.

Missirlis, K. A., Assimacopoulos, D. and Mitsoulis E., 1998, A finite volume approach in the simulation of viscoelastic expansion flows, Journal of Non-Newtonian Fluid Mechanics, Vol. 78, pp. 91118. 
Pinho, F. T., Oliveira, P. J. and Miranda, J. P., 2003, Pressure losses in the laminar flow of shearthinning power-law fluids across a sudden axisymmetric expansion, International Journal of Heat and Fluid Flow, Vol. 24, pp. 747-761.

Ternik, P., Marn, J.and Zunik, Z., 2006, NonNewtonian fluid flow through a planar symmetric expansion: Shear-thickening fluids, Journal of NonNewtonian Fluid Mechanics, Vol. 135, pp. 136-148.

Vaz Jr., M. and Zdanski, P. S. B., 2007, A fully implicit finite difference scheme for velocity and temperature coupled solutions of polymer melt flow, Communications in Numerical Methods in Engineering, Vol. 23, pp. 285-294.

Zdanski, P. S. B. and Vaz Jr., M., 2006a, Polymer melt flow in plane channels: effects of the viscous dissipation and axial heat conduction, Numerical Heat Transfer Part A: Applications, Vol. 49, No. 2, pp. 159-174.

Zdanski, P. S. B. and Vaz Jr., M., 2006b, Polymer melt flow in plane channels: hidrodynamic and thermal boundary layers, Journal of Materials Processing Technology, Vol. 179, pp. 207-211.

Zdanski, P. S. B., Ortega, M. A. and Fico Jr., N. G. C. R., 2004, Numerical simulation of the incompressible Navier-Stokes equations, Numerical Heat Transfer Part B: Fundamentals, Vol. 46, No. 6, pp. 549-579.

Zdanski, P. S. B., Ortega, M. A., Fico Jr., N. G. C. R., 2005, Heat transfer studies in the flow over shallow cavities, Journal of Heat Transfer, Vol. 127, pp. 699-712. 